\title{
ROMANIAN
}

NEUROSURGERY

\author{
Vol. XXXIV | No. 2 June 2020
}

Predictors of severe head injury in

tertiary centre

Rajendra Shrestha 


\title{
Predictors of severe head injury in tertiary centre
}

\section{Rajendra Shrestha}

\author{
Department of Neurosurgery, National Academy of Medical Sciences, \\ National Trauma Centre, Bir Hospital, Kathmandu, NePAL
}

\begin{abstract}
Introduction: Severe head injury (SHI) is associated with a high mortality and morbidity rate and is one of the leading causes of death in intensive care units. The aim of this study was to identify predictors of hospital outcome and mortality in ICU admitted SHI patients and to estimate their impact.

Methods: A retrospective analysis was carried on patients $(n=321)$ with a severe head injury, defined as Glasgow Coma Scale (GCS) $\leq 8$ who were admitted to the ICU neurosurgical department of National Trauma Center from 2017 to 2018. Both clinical and radiological predictors of hospitalized patients were identified.

Results: Total mortality rate was 5.9\%. 243 (75.7\%) of the patients were male and 78 (24.3\%) were female. $55 \%$ of cases were due to traffic accidents. Patients Middle Ages group was $60 \%$. Coexisting injuries, found in $25 \%$ of the patients aggravated the prognosis. Blood grouping pattern in $\mathrm{SHI}$ were $\mathrm{B}+, \mathrm{A}+$ and $\mathrm{O}+36.1,28$ and $24.3 \%$ respectively. $45 \%$ of the patients had Tattoo which was aggravating factors. The outcome is highly correlated with GCS' values. CT scan findings revealed that patients with subdural hygroma after few days of admission CT scan which was very important prognostic factors in SHI.

Conclusions: SHI has high mortality and morbidity in today world as it has a high negative impact on young people, especially men with blood group $\mathrm{B}+$. The age of the patient, presence of Tattoo, GCS at admission, the CT scanning at admission and CT scanning after a week of admission were significant predictors of outcome.
\end{abstract}

\section{INTRODUCTION}

Head injury (HI) is injuries to the scalp, skull, or brain (Traumatic Brain injury) produced by trauma, possibly leading to permanent or temporary impairment of cognitive, physical, and psychosocial functions, with diminished or altered state of consciousness. Severe traumatic brain injury (TBI) is associated with a high mortality and morbidity rate and is one of the leading causes of death in the intensive care units $(3,16)$. This is a retrospective study to identify predictors of hospital outcome/ mortality in ICU admitted severe TBI patients and to estimate their impact. Moreover, this study also intends to find the recent trends of head injury.

\section{MATERIALS AND METHODS}

A retrospective analysis was done on patients with severe head injury,

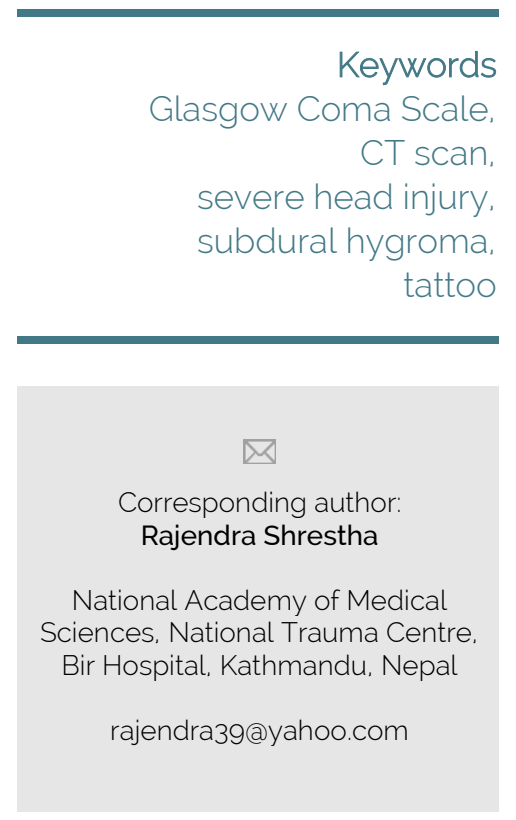

Copyright and usage. This is an Open Access article. distributed under the terms of the Creative Commons Attribution Non-Commercial No Derivatives License (https://creativecommons org/licenses/by-nc-nd/4.0/) which permits noncommercial re-use, distribution, and reproduction in any medium, provided the original work is unaltered and is properly cited.

The written permission of the Romanian Society of Neurosurgery must be obtained for commercial re-use or in order to create a derivative work.

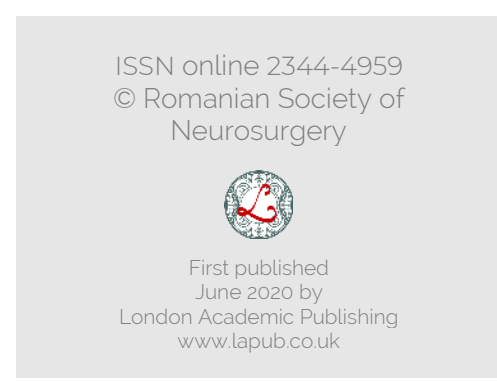


defined as Glasgow Coma Scale (GCS) $\leq 8$, who were admitted to the ICU neurosurgical department of National Trauma Center from June 2017 to July 2018. CT scan brain plain was done as a part of routine evaluation for all patients when presented in emergency department (ER). Patient's inpatient files, ER and police records were used to retrieve the data. The consciousness level was assessed by the Glasgow Coma Scale (GCS) and the outcome was assessed by the Glasgow Outcome Score (GOS). Patient characteristics like age, gender distribution, mode of injury, alcohol influence, GCS on admission, pupil reactivity, presence of extra-cranial associated injuries, laboratory tests and CT scan results were evaluated.

Outcome assessment for survivors was based on data from the medical records of patients during their hospitalization, and that obtained within a 6 month-period after discharge. Unfavorable outcome (death or severe disability) at six months was defined with the GOS. The scale comprises five categories: death, vegetative state, severe disability, moderate disability, and good recovery. Our results are statistically analyzed and discussed. Inclusion criteria: All the severe head injury patients who were admitted in intensive care unit under the Neurosurgery care during the study period. Exclusion criteria: The severe head-injured patients who had polytrauma with cervical injury.

\section{RESULTS}

In this study, there were 321 patients with severe head injury. $243(75.7 \%)$ of the patients were male and $78(24.3 \%)$ were female (Figure 1). Patients Middle Ages group was $60 \%$ (Figure 2). The number of patients with severe head injury admitted to the hospital was 321, out of which 174 (54\%) patients had a head injury following Road traffic accident (RTA), 114 (36\%) sustained head injury following fall from height, 20 (6\%) secondary to physical assault, and 13 (4\%) attributed to other modes of injury (Figure 3). Most of the cases admitted in ICU (96\%) were intubated and mechanically ventilated. The radiological findings on head injury patients showed contusion in most cases around 29\% (Figure 4). Nonsurgical (conservative) treatment was provided to 225 (70\%) patients while 96 (30\%) patients required surgical intervention (Figure 5). Coexisting injuries, found in $25 \%$ of the patients, affected the prognosis. Two third of the patients admitted with SHI were under the influence of alcohol (Figure 6). Blood grouping pattern in $\mathrm{SHI}$ were $\mathrm{B}+\mathrm{A}+$ and $\mathrm{O}+36.1,28$ and $24.3 \%$ respectively (Figure 7 ). $45 \%$ of the RTA patients had Tattoo which was aggravating factors. CT scan findings revealed that patients with subdural hygroma after few days of admission CT scan which was very important prognostic factors in TBI. Outcome highly correlated with GCS score.

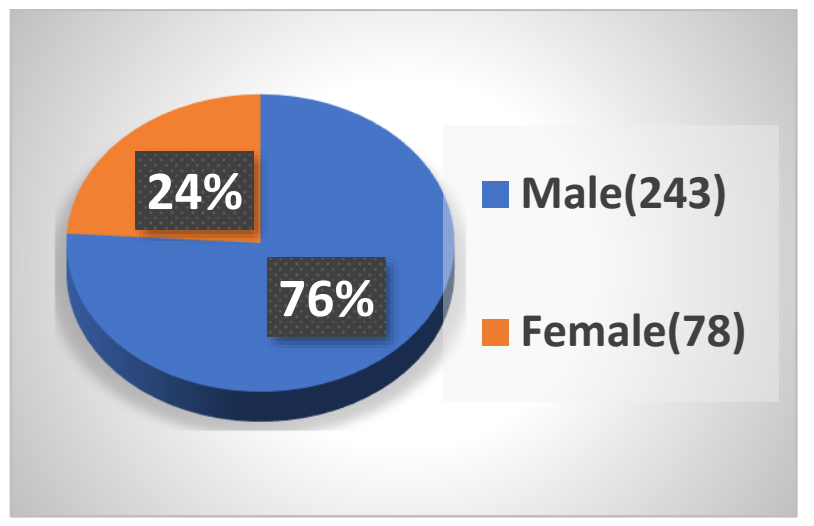

Figure 1. Gender distribution of admitted SHI.

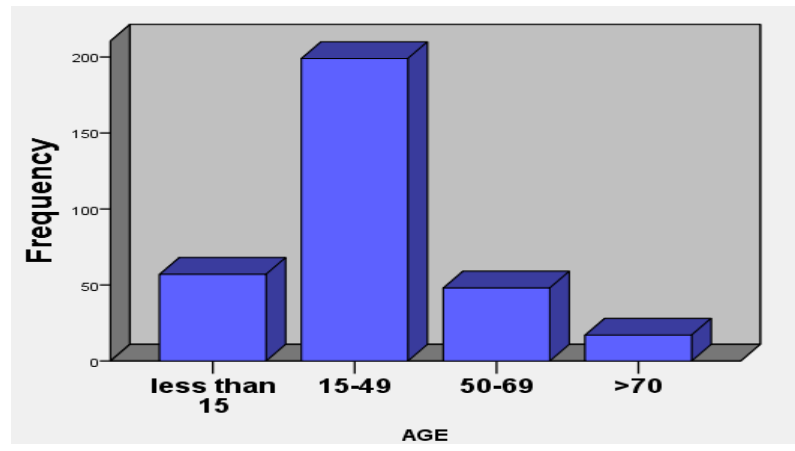

Figure 2. Age distribution of admitted SHI.

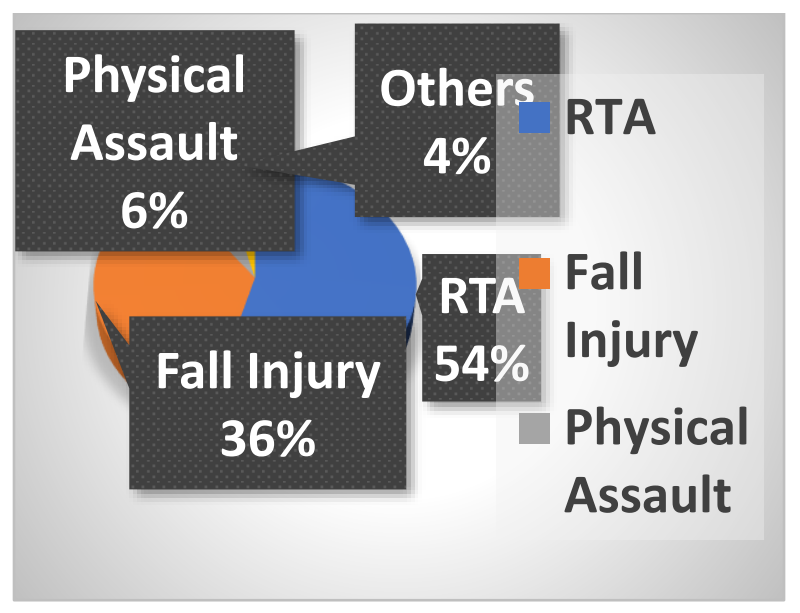

Figure 3. The distribution of causes of injury in admitted SHI $(n=321)$. 


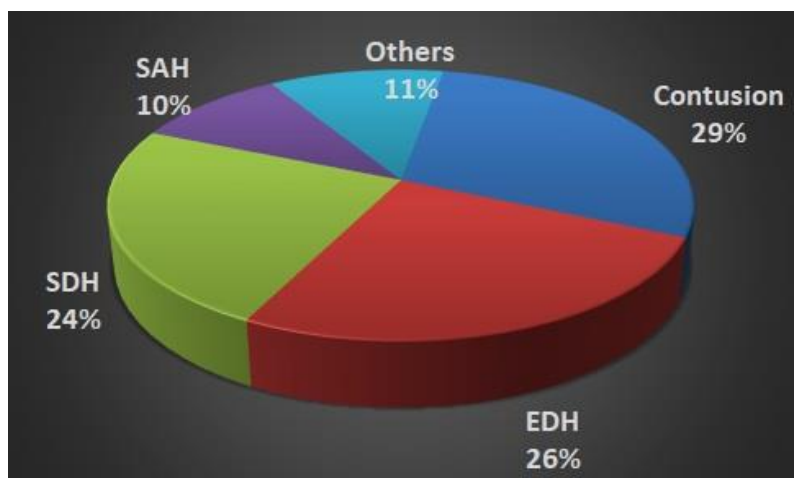

Figure 4. Radiological Finding of admitted SHI patients.

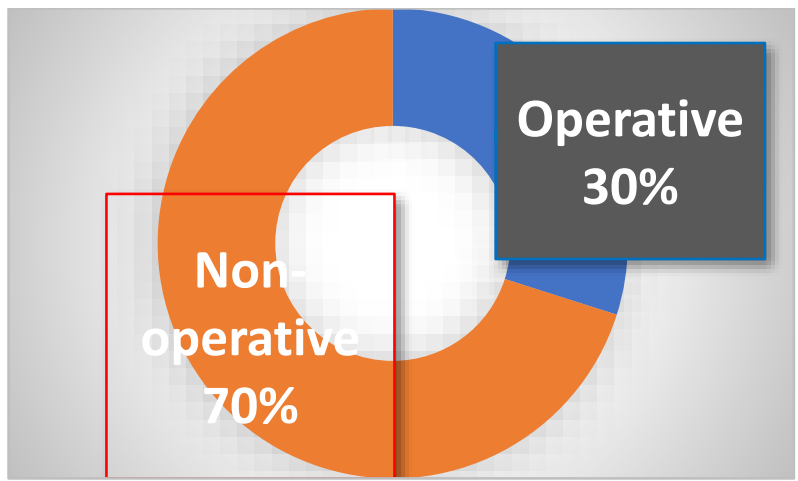

Figure 5. Management of admitted SHI patients.

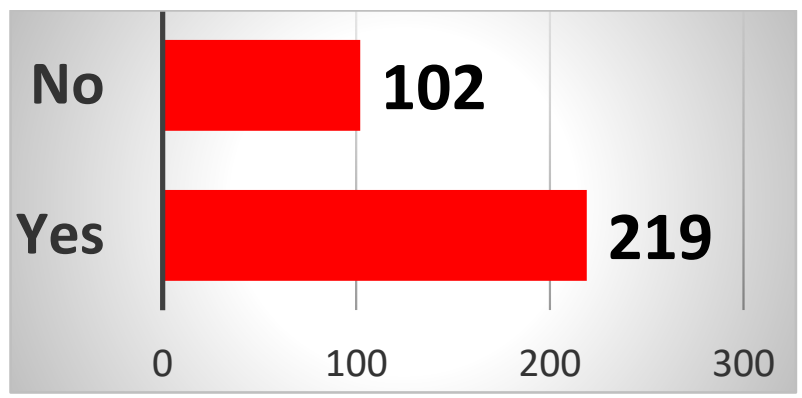

Figure 6. Numbers of patients under alcohol influences.

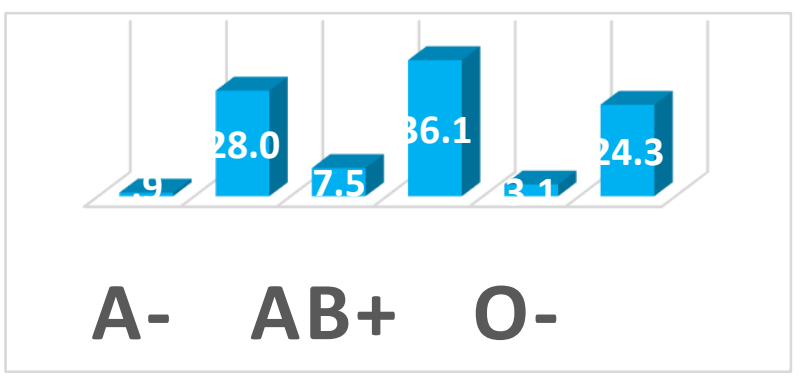

Figure 7. Blood grouping pattern in Severe Head injuries.

\section{Discussion}

Severe head injury (SHI) is the most common global cause of morbidity and mortality in people under 45 years of age and possess major public health and socioeconomic challenges, which can negatively impact the daily life activities and possess high risk of readmission to hospital and subsequent death. Despite advancements in diagnosis and treatment, there are many queries regarding the diagnosis, prognosis and best possible treatment of SHI. There are no acceptable clinical and pathological predictive factors have been recognized for developing clinical trials and promoting effective therapeutic strategies to improve the prognosis. There were a lot of different prognostic factors related to outcome. The most important prognostic factors included: age, Glasgow Coma Scale, pupil size, coexistence of other injuries, a history of previous head injury, alcohol abuse and lower socioeconomic, educational status, hypotension, hypoxia, glucose, coagulopathy, haemoglobin, Marshall CT classification and traumatic subarachnoid haemorrhage $(1,2,9$, and 11). This article focuses mainly on the clinically significant aspects of potential interrelationship between potential diagnostic and prognostics tools in severe brain injury. Assessing prognosis after traumatic brain injury was both very important and difficult.

In this study, the majority of patients were male $76 \%$ and only $24 \%$ were female (Figure 1 ). Patients of both sexes primarily belong to an age group of 15-50 years in the age-distribution curve of SHI (Figure 2). This could be credited to this group of people predominantly being more active and vulnerable in the community and country. In the present study, RTA was established as the most common cause of SHI. It contributed to almost $55 \%$ of the head injury cases leading to admission (Figure 3 ). This finding can be attributed to more men being drivers and more prone to be involved in RTA $(5,6)$. Noticeably, cases of head injuries due to the physical assault appear to be on the rise, compared to zero percent in the study of Gongal and Devkota (1979) to 6\% in the present series (4).

In Nepal, the zero-tolerance policy against drinking and driving significantly reduced the number of RTA in past years. However, two third of the patients with $\mathrm{SHI}$, in this study were under the influence of alcohol. Patients with SHI with GCS $\leq 8$ were intubated and mechanically ventilated in ICU. Nearly all patients in the present study underwent CT imaging of the head immediately after emergency admission. Neurosurgical procedures were 
performed between admission to the emergency room and transfer to intensive care unit.

Initial CT examination demonstrated abnormalities in approximately $90 \%$ of patients with severe head injury. CT scan plays a vital role in early assessment of patients with severe head injury. Repeat CT scan was done within 48-72 hours to exclude conversion of contusion into increase hematoma. Our study showed, patients with SHI developed subdural hygroma after a week, as seen on their CT scans. Subdural hygroma is defined as acute or chronic cerebrospinal fluid accumulation in the subdural space, which is a virtual space between the arachnoid and subdural membranes (17). In our series, bilateral subdural hygroma were located mainly frontoparietal region representing more than $77 \%$ of the cases. In the literature, this sort of lesion is usually related with contusions, cerebral atrophy, subdural and epidural hematomas or post operatory complications (12, 15, and 17). Although etiology is well documented, pathogenesis of this condition is not well explained. Some author proposed that the formation of a one-way valve allowing the passage of CSF into the subdural space, caused by a traumatic tear of the arachnoidal mater or rupture of incidental arachnoids cyst $(7,13)$. In our series, we noticed prognosis of patients could be good once bilateral subdural hygroma in the CT scan.

The study also revealed a correlation between tattoo and SHI. $45 \%$ of RTA cases with SHI had tattoo. However, present research does not indicate any particular group, age, gender, occupation, personality or a particular level of social status that get tattoos. There are many reasons to why people get tattoo; describing personal tales, expressing individuality, spiritual meaning, fashion, scar hiding, marking new beginnings like birth, adulthood, marriage, divorce, death, etc. and even marking memories as milestone for love or loss (14).

Another interesting finding of our study, Blood grouping pattern in $\mathrm{SHI}$ were $\mathrm{B}+, \mathrm{A}+$ and $\mathrm{O}+36.1,28$ and $24.3 \%$ respectively (Figure 7 ). In our country, normal blood grouping distribution are $\mathrm{A}, \mathrm{O}, \mathrm{B}$ and $A B$ at $55.05 \%, 21.64 \%, 14.72 \%$, and $8.6 \%$ respectively (8). But $O, A, B$ and $A B$ were $46 \%, 41 \%, 9 \%$ and $4 \%$ in the USA respectively (10). These findings can be incidental or crucial. Further study needs to be conducted as our study had its limitation; small study group, single center findings and limited follow up to understand its outcome. We strongly recommended that prediction factors should be kept in mind for the best management and outcome of severe head injury patients. Head trauma should highly be prevented in order to reduce incidence of TBI related mortality. Thus, awareness of the impact of head trauma should be raised through information campaigns.

\section{CONCLUSION}

Severe head injury has a high negative impact on young people, especially men and also B+ blood group. The age of the patient, presence of Tattoo, GCS at admission, CT scanning at admission and CT scanning after week of admission with hygroma were significant predictors of outcome.

\section{REFERENCES}

1. Baguley IJ, Nott MT, Howle AA, Simpson GK, Browne S, et al. Late mortality after severe traumatic brain injury in New South Wales: a multi center study. Med J Aust, 2012; 196: 40-45.

2. Brazinova A, Rehorcikova V, Taylor MS, Buckova V, Majdan $M$, et al. Epidemiology of Traumatic Brain Injury in Europe: A Living Systematic Review. Journal of Neurotrauma 2016; 33:1-30.

3. Cesar Reis et al. What's New in Traumatic Brain Injury: Update on Tracking, Monitoring and Treatment; International Journal of Molecular Science,2015; 16: 11903-11965

4. Gongol DN, Devkota UP. An analysis of head injuries in children of Nepal. Journal of Nepal Medical Association (JNMA Souvenir): 1979;181-9.

5. Husson EC, Ribbers GM, Willemse-van Son AH, Verhagen $A P$, Stam HJ, et al. Prognosis of six-month functioning after moderate to severe traumatic brain injury: a systematic review of prospective cohort studies. J Rehabil Med, 2010; 42: 425-436.

6. Kolias AG, Guilfoyle MR, Helmy A, Allanson J, Hutchinson PJ:Traumatic brain injury in adults. Pract Neurol,2013; 13: 228-235.

7. Kusuno, K., Yoshida, Y., Takahashi, A., \& Ishii, S.: Chronic subdural hygroma caused by rupture of arachnoid cyst. As a probable course of chronic subdural hematomacase report. Neurologia Medico-Chirurgica, 1984; 24(5), 349-354. http://dx.doi.org/10.2176/nmc.24.349.

8. Lava Shrestha, uzwali malla:ABO and Rh Blood Groups and their Ethnic Distribution in a Teaching Hospital of Kathmandu, Nepal ,2013; Vol 52 190. DOI: https://doi.org/10.31729/jnma.2112

9. Maas Al, Steyerberg EW, Butcher I, Dammers R, Lu J, Marmarou A, et al. Prognostic Value of Computerized Tomography Scan Characteristics in Traumatic Brain Injury: Results from the IMPACT Study. J Neurotrauma. 2007; 24(2): 303-314. 
10. Mollison PL, Engelfriet CP, Conteras M. The Rh blood Group system. In Blood Transfusion in Clinical Medicine, 9th Edition. Oxford: Black well Scientific Publication.1993; 2008-09.

11. Murray GD, Butcher I, McHugh GS, Lu J, Mushkudiani NA, Maas Al, et al. Multivariable prognostic analysis in traumatic brain injury: results from the IMPACT study. J Neurotrauma 2007; 24(2): 329-337.

12. Park, J., Cho, J.-H., Goh, D.-H, et al. Postoperative Subdural Hygroma and Chronic Subdural Hematoma after Unruptured Aneurysm Surgery: Age, Sex, and Aneurysm Location as Independent Risk Factors. Journal of Neurosurgery, 2015; 1-8.

13. Pillai, P., Menon, S. K., Manjooran, R. P., Kariyattil, R., Pillai, A. B., \& Panikar, D. Temporal Fossa Arachnoid Cyst Presenting with Bilateral Subdural Hematoma Following Trauma: Two Case Reports. Journal of Medical Case Reports, 2009; 3(1), 53. http://dx.doi.org/10.1186/17521947-3-53.
14. Shannon Bell;Tattooed: A Participant Observer's Exploration of Meaning,The Journal of American Culture,2004; 22(2):53-58:DOI: 10.1111/j.1542734X.1999.2202_53.x.

15. Sun, H.-L., Chang, C.-J., \& Hsieh, C.-T. Contralateral Acute Subdural Hematoma Occurring after Evacuation of Subdural Hematoma with Coexistent Contralateral Subdural Hygroma. Neurosciences (Riyadh, Saudi Arabia), 2014:19(3), 229-232.

16. Traumatic Brain Injury-Definition, Epidemiology, Pathophysiology: Segun Toyin Dawodu, Updated Aug 16,2017; $\quad$ https://emedicine.medscape.com /article/326510-overview.

17. Zanini, M. A., Resende, L. A. D. L., Faleiros, A. T. D. S., \& Gabarra, R. C.Traumatic Subdural Hygromas: Proposed Pathogenesis Based Classification. The Journal of $\begin{array}{llll}\text { Trauma, } 2008 ; & 64 & \text { (3), }\end{array}$ ttp://dx.doi.org/10.1097/TA.0b013e3180485cfc. 